\title{
A Space-Efficient and Self-Stabilizing Depth-First Token Circulation Protocol for Asynchronous Message-Passing Systems * \\ (Short Version)
}

\author{
Franck Petit and Vincent Villain \\ LaRIA, Université de Picardie Jules Verne \\ 5, rue du Moulin Neuf F - 80039 AMIENS Cedex 1 FRANCE
}

\section{Introduction}

Self-Stabilization was first introduced by Dijkstra in [Dij74]. In this pioneering paper, Dijkstra defines a system self-stabilizing if, starting with an arbitrary initial state, the system is guaranteed to reach a legitimate state in a finite number of steps. The self-stabilization property is very useful for distributed systems in which transient failures may occur and recover in an arbitrary state.

In [CH93], Huang and Chen present the first self-stabilizing depth-first token circulation for general networks. The space complexity of their algorithm depends on $n, n$ is the number of processors. In [BJ95], Johnen and Beauquier have improved the [CH93] result by reducing space for each processor to the best known complexity, i.e. $\mathcal{O}(\log (\Delta)), \Delta$ is the upper bound of node's degree. Both algorithms run on state model far enough from message-passing model, the most realistic model. In asynchronous message-passing systems, transient failures may damage local variables and the content of communications links.

Using the algorithm developed in [AB93], we transform the algorithm shown in [BJ95] to obtain the first self-stabilizing depth-first token circulation for asynchronous message-passing system. Such an algorithm is very desirable to solve many problems in arbitrary message-passing system, e.g. mutual-exclusion, spanning-tree, synchronization or to make implementable self-stabilizing automatic transformation of arbitrary distributed algorithms [FV97]. Moreover, we keep the same advantages as in [B.J95], i.e. space complexity depends on $\Delta$ instead of $n$. This property is very useful because in many networks $\Delta \ll n$ and since the algorithm only needs localy properties, it runs on dynamic networks in which the topology may change during the execution. When the system is stabilized, the message complexity is $\mathcal{O}(m \Delta)$ for the complete round, in which $m$ is the number of communications links in the network.

* This research was supported in part by the Pole of Modelization of Picardie, France. Correctness and full paper are available at <http://www.laria.u-picardie.fr $>$ or by Email: <\{petit,villain\}@laria.u-picardie.fr $>$. 


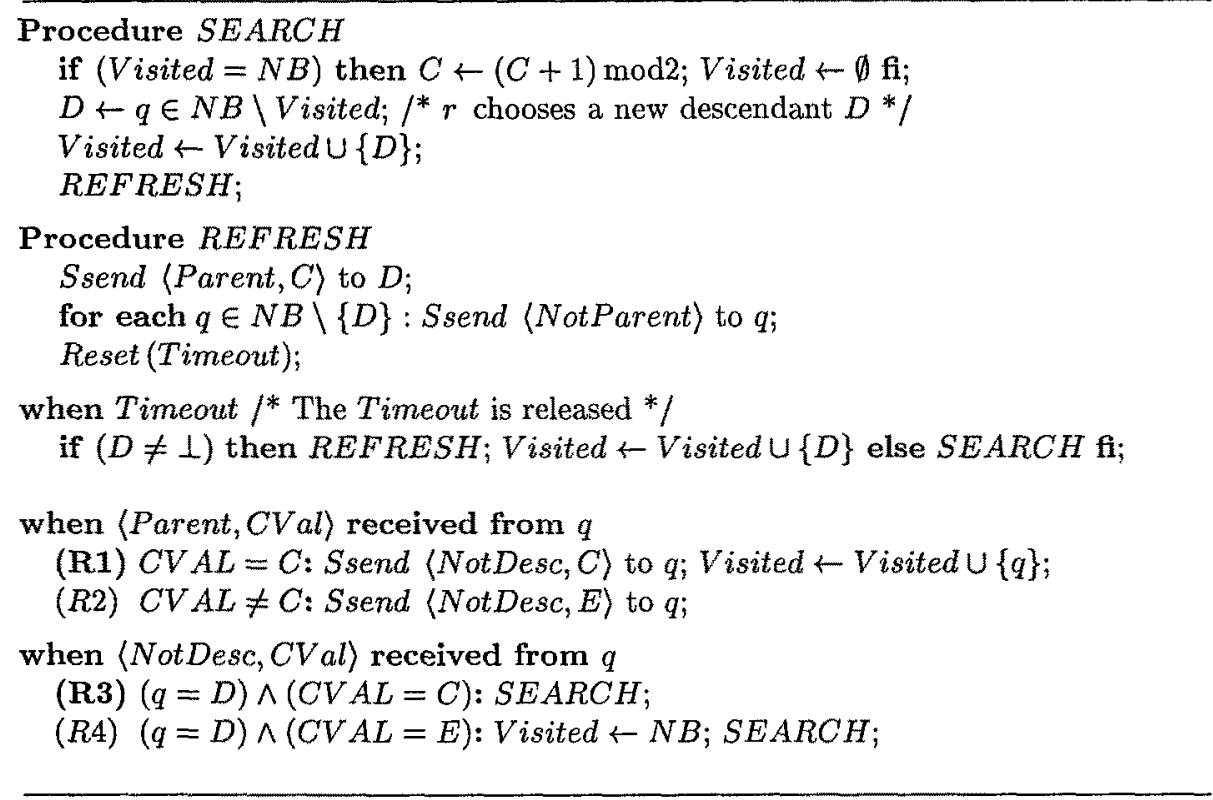

Fig. 1. The Root algorithm

\section{Description of the Protocol}

The system consists of a connected network. Nodes are processors with no distinct identities except one called the root $(r)$. Edges are undirected communication links. Each processor is hard-wired with communication links and communicates with its neighbors by sending messages which arrive in the order they have been sent (FIFO). The system stabilizes if starting from an arbitrary configuration, it eventually reaches the legitimate region $\mathcal{L S}$ in which the protocol infinitely makes circulate a unique token in the depth-first search order.

The protocol is organized into two distinguished levels. First, the low level corresponds to the self-stabilization of the links which is an adaptation to our problem of the Self-Stabilizing Alternating Bit Protocol shown in the [AB93] paper. Secondly, the application level describes as follow.

Pointers on links are stored in the set $N B$ which can not be corrupted. Each processor $p$ different from the root $r$ maintains the following variables: $C$ is the round color which can take value into the set $\{0,1, E\}, D$ and $A$ are components of $N B$, respectively the unique descendant and ascendant of $p$ ( $\perp$ if $D$ or $A$ does not exist) and $V$ isited is a subset of $N B$ which contains the neighbors that $p$ consider like visited. The root $r$ maintains the same variables except $C$ which takes its value into $\{0,1\}$ and $A$ because the root does not have any ascendant.

To make the token circulate, we use three types of messages. Both of them contain the round color value $C V$ al: the $\langle$ Parent, $C V$ al $\rangle$ message sent by a processor to its descendant and the $\langle N$ otDesc, $C V$ al $\rangle$ message sent by a processor 
when $\langle$ Parent, $C V a l\rangle$ received from $q$

$$
\begin{aligned}
& (A=\perp) \wedge(D=\perp) \wedge(C \neq E) \\
& \wedge(C V a l \neq C) \wedge(C V a l \neq E): A \leftarrow q ; C \leftarrow C V a l
\end{aligned}
$$

$/^{*} p$ is reached by a new ascendant $* / V$ isited $\leftarrow\{A\} ; S E A R C H$;

$$
\begin{aligned}
& (A \neq 1) \wedge(A \neq q) \wedge(D \neq \perp) \\
& \wedge(C \neq E) \wedge(C \vee a l=C): C L E A R(q, C V a l)
\end{aligned}
$$

$(R 7)((A=\perp) \wedge(D=\perp) \wedge(C \neq E) \wedge(C \vee a l=C))$

$/^{*} p$ is already reached $* / V i s i t e d \leftarrow V i s i t e d \cup\{q\}$;

$\vee((A \neq \perp) \wedge(A \neq q) \wedge(D \neq \perp) \wedge(C \vee a l=E)): C L E A R(q, C V a l)$;

$f^{*} p$ is not reachable by this search $* /$

(R8) $(A=\perp) \wedge(D=\perp) \wedge((C=E) \vee(C V a l=E)): C L E A R(q, C V a l) ; C \leftarrow 0 ;$

$f^{*} p$ is path-free, not reachable by this search $*$ )

(R10) $\quad(A \neq \perp) \wedge(A=q) \wedge(D \neq \perp) \wedge(C V a l=E)$ : Ssend (Parent, $E)$ to $D$;

$/^{*} p$ propagates the color $E^{*} / C \leftarrow E$;

$$
(A \neq \perp) \wedge(A \neq q) \wedge(D \neq 1)
$$$$
\wedge(C V a l \neq E) \wedge(C V a l \neq C): C L E A R(A, E) ; A \leftarrow q ;
$$

$l^{*}$ 2nd search: $q$ is the new asc., $p$ is $E$-colored* $/ C \leftarrow E$;

$$
(A \neq \perp) \wedge(D=\perp) \text { : for each } q^{\prime} \in\{A, q\}
$$

$$
\text { CLEAR }\left(q^{\prime}, E\right)
$$

$I^{*}$ illegal leaf detection: $p$ becomes path-free ${ }^{*} / A \leftarrow \perp ; C \leftarrow 0$;

when $\langle$ NotParent $\rangle$ received from $q$

$$
\begin{gathered}
(A=q) \wedge(D \neq \perp): A \leftarrow L ; C \leftarrow E \\
I^{*} p \text { becomes an illegal root } E \text {-colored } * / \\
(A=\perp) \wedge(D \neq \perp): C \leftarrow E ; \\
/^{*} p \text { is an illegal root } * / \\
(A=\perp) \wedge(D=\perp) \wedge(C=E): C \leftarrow 0 ; \\
/^{*} p \text { is path-free and not reachable by this search } * / \\
(A \neq \perp) \wedge(D=\perp): C L E A R(A, E) ; \\
/^{*} \text { illegal leaf detection: } p \text { becomes path-free } * / C \leftarrow 0 ;
\end{gathered}
$$

when $\langle$ NotDesc, CVal $\rangle$ received from $q$

$(R 18) \quad(A \neq \perp) \wedge(q=D) \wedge((C V a l=E) \vee(C=E)): C L E A R(A, E)$;

$/^{*} p$ 's desc. search completed and $E$-colored $* / A \leftarrow \perp ; D \leftarrow \perp ; C \leftarrow 0$;

(R19) $(A=\perp) \wedge(q=D) \wedge((C V a l=E) \vee(C V a l=C)): D \leftarrow \perp ; C \leftarrow 0$;

$/^{*} p$ is an illegal root and its search is completed $*$

(R20) $\quad(A=\perp) \wedge(D \neq \perp) \wedge(C \neq E) \wedge(q \neq D): C \leftarrow E$

$/^{*} p$ is an illegal root and has to be $E$-colored $*$

$$
(A=\perp) \wedge(D=\perp) \wedge(C=E): C \leftarrow 0
$$

$f^{*} p$ is path-free, not reachable by this search */

$(A \neq \perp) \wedge(D=\perp): C L E A R(A, E) ; A \leftarrow \perp ;$

$/^{*}$ illegal leaf detection: $p$ becomes path-free $* / C \leftarrow 0$;

Fig. 2. The Not Root event reception 


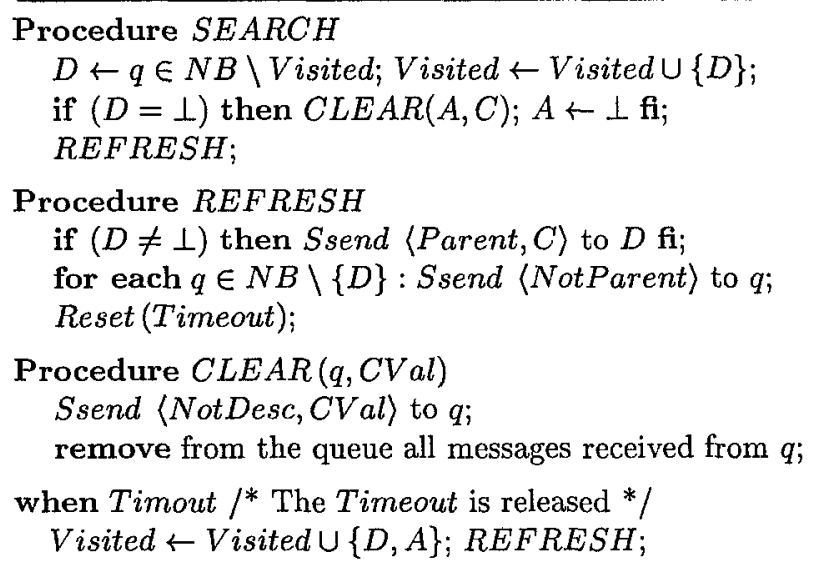

Fig. 3. The Not Root procedures

to make its ascendant know the end of the visit. The third one is $\langle N$ otParent $\rangle$ broadcasted by each processor to all its neighbors except its descendant to recover correct relationship between processors.

The algorithm, fig. 1 and fig. 2, consists of a collection of rules ( $\langle$ guard $\rangle$ : $\langle$ action $\rangle)$ locally evaluated when a message is received by a processor. The rules use some procedures described fig. 1 and fig. 3 . Bold rules numbers indicate the rules of the basic algorithm. Other rules are the error handling in which bad paths (cycles and paths not rooted by $r$ ) are destroyed by $E$-coloration.

To make sure that the top level protocol of each processor infinitely often sends a message to its neighbors, both self-stabilizing function Timeout and $R E F R E S H$ procedure ensure the infinity of messages. Moreover, to avoid tokens

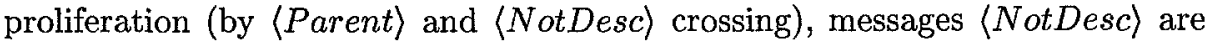
processed with high priority and the reception of a $\langle N o t D e s c\rangle$ acknowledgement cleans messages of the corresponding neighbor from the queue. (CLEAR()).

\section{References}

[AB93] Afek Y. and Brown G.M., "Self-Stabilization Over Unreliable Communication Media", Distributed Computing 7, 1993, pp.27-34.

[BJ95] Johnen C. and Beauquier J., "Space-efficient, Distributed and Self-Stabilizing Depth-First Token Circulation", Proc. 2th Workshop on Self-Stabilizing Systems, Las Vegas, May 1995, p 4.1-4.15.

[CH93] Chen N-S and Huang S-T., "Self-Stabilizing Depth-First Token Circulation on Networks", Distributing Computing 7, 1993, pp. 61-66.

[Dij74] Dijkstra E.W., "Self-Stabilizing Systems in Spite of Distributed Control", Communications of the ACM 17, 1974, pp. 643-644.

[FV97] O Flauzac and V Villain. An implementable dynamic automatic self-stabilizing protocol. RR 97-06, LaRIA, University of Picardie Jules Verne, 1997. 